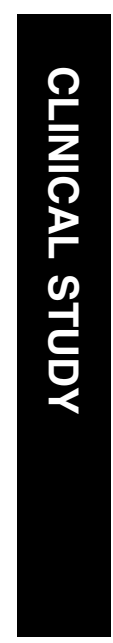

\section{Inhibitory effects of maternal smoking on the development of severe retinopathy of prematurity}

${ }^{1}$ Division of Ophthalmology, Department of Surgery, Kobe University Graduate School of Medicine, Hyogo, Japan

2Department of Pediatrics, Kobe University Graduate School of Medicine, Hyogo, Japan

${ }^{3}$ Department of EvidenceBased Laboratory Medicine, Kobe University Graduate School of Medicine, Hyogo, Japan

Correspondence: S Honda, Division of Ophthalmology, Department of Surgery, Kobe University Graduate School of Medicine, 7-5-2 Kusunoki-cho, Chuo-ku, Kobe, Hyogo 650-0017, Japan

Tel: + 8178382 6048;

Fax: + 8178382 6059;

E-mail: sighonda@

med.kobe-u.ac.jp

Received: 3 December 2008 Accepted in revised form: 21 September 2009

Published online:

6 November 2009

\begin{abstract}
Purpose In this study, the effects of maternal smoking along with other clinical risk factors in developing severe retinopathy of prematurity (ROP) were evaluated.

Design A case-control study.

Methods Records of newborn infants with an estimated postmenstrual age of 32 weeks or less $(n=86)$ were reviewed. ROP grading was evaluated in accordance with the International Classification of Retinopathy of Prematurity. Severe ROP was diagnosed when it progressed to stage 3 with plus disease. The factors were first evaluated using a univariate logistic regression analysis between the groups of severe and non-severe ROP, followed by a multivariate logistic regression analysis using STATA version 10 and $R$ version 2.71 .
\end{abstract}

Results A low birth weight, a long duration of artificial ventilation and oxygen supplementation, presence of chronic lung disease, and absence of maternal smoking were found to be significantly associated with severe ROP in the univariate logistic regression analysis. In the multivariate logistic regression analysis, maternal smoking was revealed as a significant factor independently associated with the incidence of severe ROP.

Conclusions An inhibitory effect of maternal smoking against developing severe ROP is suggested. The mechanism by which smoking may reduce the incidence of severe ROP needs to be further investigated.

Eye (2010) 24, 1024-1027; doi:10.1038/eye.2009.263; published online 6 November 2009

Keywords: retinopathy of prematurity; risk factor; statistical analysis; smoking
H Hirabayashi', S Honda', I Morioka², N Yokoyama ${ }^{2}$, D Sugiyama ${ }^{3}$, K Nishimura ${ }^{3}$, $M$ Matsuo ${ }^{2}$ and $A$ Negi'

\section{Introduction}

Retinopathy of prematurity (ROP) is a serious disorder in premature infants. Although most cases resolve spontaneously, some cases progress to fibrovascular proliferation and tractional retinal detachment, which result in a poor visual prognosis. ${ }^{1}$ Preventing severe ROP is the most important issue in the ophthalmic care of preterm infants. Recent reports have documented the risk factors for severe ROP, which include low postmenstrual age, low birth weight, excessive oxygen supplementation, and male gender. ${ }^{2-5}$ However, the influence of maternal cigarette smoking on severe ROP has not been fully understood to date.

Maternal smoking during pregnancy has been causally associated with adverse pregnancy outcomes, including placental abruption, placenta previa, preterm delivery, intrauterine growth retardation, and fetal death. ${ }^{6-8}$ It is also associated with postnatal infections in preterm neonates. ${ }^{9}$ Therefore, we hypothesized that maternal smoking might be a risk factor for ROP. In addition, as the prevalence of maternal smoking is estimated at about 18-20\% in Japanese society, investigating the effect of smoking on the development of ROP is an important issue. ${ }^{10}$

In this study, we performed a statistical evaluation on clinical risk factors including maternal smoking for the incidence of severe ROP in the Japanese population.

\section{Materials and methods}

The records of newborn infants with an estimated postmenstrual age of 32 weeks or less between January 2005 and June 2007 at the mother and baby centre of Kobe University 
were retrospectively reviewed in this study. The first ophthalmic examination was performed at either 3-4 weeks of age or at 31 weeks of gestation, which was earlier, and followed up until a postmenstrual age of 40 weeks. The classification of ROP was determined according to the International Classification of Retinopathy of Prematurity. ${ }^{11}$ The diagnosis of severe ROP was made on the basis of (1) the presence of stage 3 plus disease in Zone 1 or 2, extending to 5 or more contiguous or 8 cumulative clock hours; or (2) severe stage 2 ROP with plus disease. The history of cigarette smoking was self-reported by each mother, and recorded in the maternal and infant medical records at the first visit to the mother and baby centre of Kobe University. ${ }^{10}$ Women who did not smoke at any time during pregnancy were included in the group of non-smokers. Women who continued to smoke during pregnancy with any frequency were included in the group of smokers. Women who discontinued smoking during pregnancy or women who had an uncertain status of smoking were not included in this study.

For statistical analysis, each clinical factor was first evaluated with univariate logistic regression analysis between the severe and non-severe ROP groups. Subsequently, a multivariate logistic regression analysis by the stepwise method was performed to evaluate the independent risk factors of severe ROP. The examined factors included gender, postmenstrual age, birth weight, singleton or multiple birth, duration of artificial ventilation including continuous positive air pressure (CPAP), total duration of oxygen supplementation, and the presence or absence of chronic lung disease (CLD) and apnoea attacks, which are known or speculated to be risk factors for severe ROP. We also evaluated maternal smoking as an additional risk factor in this study. We used STATA version 10 (StataCorp, Lakeway Drive College Station, Texas,
USA) and R version 2.71 ( $\mathrm{R}$ Foundation for Statistical Computing, Vienna, Austria) for all statistical analyses. $P$-values of 0.05 or less were considered to be statistically significant.

\section{Results}

A total of 86 infants (male: 46 infants, female: 40 infants) were included in this study. The mean postmenstrual age of the subjects was 28.9 (SD 2.5) weeks, and the mean birth weight was 1108.8 (SD 346.3) grams. Severe ROP developed in 27 cases (31.4\%).

The ratio of maternal smokers was about $18.6 \%$ (16 cases) of total subjects, which was very similar to that of a previous report. ${ }^{10}$ They reported smoking an average of 13.0 (range: 2-30) cigarettes per day. Birth weight, duration of artificial ventilation including CPAP, total duration of oxygen supplementation, the presence of CLD, and maternal smoking were determined to be significantly different between the severe and non-severe ROP groups by univariate logistic regression analysis (Table 1). In the multivariate logistic regression analysis, postmenstrual age, duration of artificial ventilation including CPAP, total duration of oxygen supplementation, presence of apnoea attack, and maternal smoking were determined as significant factors associated with the incidence of severe ROP (Table 2). As duration of artificial ventilation, total duration of oxygen supplementation, and maternal smoking were significantly different between the severe ROP and nonsevere ROP groups in both univariate and multivariate analyses, we determined these factors as statistically significant factors that were associated with the incidence of severe ROP.

The mean postmenstrual age and birth weight were $28.8 \pm 2.6$ weeks and $1088.8 \pm 361.9 \mathrm{~g}$ in the nonsmoking group, and $29.5 \pm 2.4$ weeks and $1196.6 \pm 293.5 \mathrm{~g}$

Table 1 Factors compared between severe and non-severe ROP groups

\begin{tabular}{|c|c|c|c|c|}
\hline & Non-severe $R O P(\mathrm{n}=59)$ & Severe $R O P(\mathrm{n}=27)$ & Odds ratio & P-value \\
\hline Number of males (\%) & $31(53)$ & $15(56)$ & 1.20 & 0.69 \\
\hline Number of multiple births (\%) & $19(32)$ & $6(22)$ & 0.60 & 0.35 \\
\hline Number of CLD (\%) & $21(36)$ & $26(96)$ & 47.05 & 0.00 \\
\hline Number of apnoea attacks (\%) & $35(59)$ & $14(52)$ & 0.74 & 0.52 \\
\hline Number of maternal smoking (\%) & $15(25)$ & $1(4)$ & 0.11 & 0.04 \\
\hline Gestational age (weeks) & $30.0 \pm 2.1$ & $26.6 \pm 1.8$ & 0.89 & 0.69 \\
\hline Birth weight $(\mathrm{g})$ & $1226.2 \pm 328.5$ & $852.4 \pm 250.8$ & 1.00 & 0.00 \\
\hline Artificial ventilation (including CPAP) (days) & $25.2 \pm 23.7$ & $61.0 \pm 18.0$ & 1.07 & 0.00 \\
\hline Oxygen supplement (days) & $35.7 \pm 23.7$ & $78.7 \pm 25.0$ & 1.07 & 0.00 \\
\hline Mother's age (years) & $32.5 \pm 4.6$ & $31.4 \pm 4.6$ & 0.95 & 0.34 \\
\hline
\end{tabular}

CLD, chronic lung disease; CPAP, continuous positive air pressure; ROP, retinopathy of prematurity.

Values represent means $\pm S D$. Statistical significances are evaluated by univariate logistic regression analysis. 
Table 2 Evaluation of independent factors associated with severe ROP by multivariate logistic regression analysis by stepwise method

\begin{tabular}{lcrrr}
\hline & OR & Z-value & 95\% CI & P-value \\
\hline Gestational age & 0.80 & -2.78 & $0.68-0.94$ & 0.01 \\
Birth weight & 1.01 & 1.85 & $1.00-1.01$ & 0.07 \\
Artificial ventilation (including CPAP) & 0.88 & -2.02 & $0.78-1.00$ & 0.04 \\
Oxygen supplement & 1.11 & 2.12 & $1.01-1.22$ & 0.03 \\
CLD & 19.65 & 2.00 & $1.06-363.41$ & 0.05 \\
Apnoea attack & 0.03 & -2.16 & $0.00-0.73$ & 0.03 \\
Maternal smoking & 0.01 & -2.33 & $0.00-0.48$ & 0.02 \\
\hline
\end{tabular}

$\mathrm{CI}$, confidence interval; $\mathrm{CLD}$, chronic lung disease; CPAP, continuous positive air pressure; OR, odds ratio.

in the smoking group $(P=0.35$ and 0.27 , respectively, using $t$-test).

\section{Discussion}

We have presented the risk factors for severe ROP in newborn infants with a postmenstrual age of 32 weeks or less. After the univariate and multivariate logistic regression analyses, maternal smoking was found to be an independent significant factor that contributes to the incidence of severe ROP. Surprisingly, it showed a potent inhibitory effect against developing severe ROP.

Lower postmenstrual age, lower birth weight, male gender, singleton birth, a larger volume of blood transfusion, and a longer duration of artificial ventilation were previously demonstrated to be associated with severe ROP. ${ }^{2-5}$ In fact, low birth weight, the presence of CLD, a long duration of artificial ventilation, and a long duration of oxygen use were demonstrated to be associated with the incidence of severe ROP in our study on the basis of univariate logistic regression analysis. However, only duration of artificial ventilation and duration of oxygen use were determined to be significant risk factors on the basis of the multivariate logistic regression analysis. The relatively small number of subjects in this study might have prevented the other variables from showing statistical significance. Interestingly, a long duration of artificial ventilation acted as a protective factor in the multivariate analysis, but it might not be clinically meaningful, as the odds ratio (0.88) was modest. As maternal smoking showed significant association with the lower incidence of severe ROP, with notable odds ratios in both the univariate and multivariate logistic regression analyses, we considered the results to be robust statistically.

Despite the insufficient assessment of the influence of maternal smoking on ROP, it is known to affect placental functions and interfere with foetal development, which is associated with small birth weight. Two reports described that maternal smoking was associated with lower birth weight ${ }^{12,13}$ and one of them showed a high rate of maternal smoking in the group of school-goingaged children with regressed ROP. ${ }^{12}$ The reason why this study did not show any difference in the mean postmenstrual age or birth weight between the smoking and non-smoking groups is unknown. We speculate that there might be different foetal or perinatal survival ratios between the smoking group and non-smoking group and only survival cases that may have a more defensive nature to smoking stress were included in this study. Recent studies reported that smoking disturbs the development of the foetal nervous system, and is associated with risks such as cognitive and auditory processing deficits. ${ }^{6-8}$ Hence, we speculate that the interference with the development of the nervous system might also affect foetal neuroretinal development, and limit the demand for oxygen. This would attenuate the stress of hypoxia, and reduce the production of angiogenic factors participating in the development of severe ROP. A recent report showed that the increased expression of vascular endothelial growth factor was associated with advanced ROP. ${ }^{14}$ Another study reported that nicotine treatment histopathologically induces marked changes in the organization of the developing retina in newborn rats. ${ }^{15}$ This enhancement of the antihypoxia defence system because of excessive intrauterine hypoxia is speculated to be another possible mechanism that reduces the incidence of severe ROP. Recent reports demonstrated an increase in haemeoxygenase 1 and 2 expressions in the placenta of smoking mothers, and increased levels of ceruloplasmin in the plasma of newborns and mothers exposed to tobacco smoking. ${ }^{16,17}$ These molecules are known as antioxidants that might reduce oxidative stress in the ischemic retina of premature infants, which might inhibit the excessive expression of angiogenic factors.

Despite the finding that maternal smoking was associated with a lower rate of severe ROP, the other effects of smoking on the consequent development of visual function remain unknown. Moreover, maternal smoking is not a recommendable behaviour because of a number of smoking-related systemic adverse events on 
mothers and infants. However, the present results may provide meaningful insights into some aspects of complicated ROP pathogenesis. Further studies are needed to elucidate the manner in which cigarette smoking or nicotine modifies the development of the fetal retina and reduces the risk of severe ROP, which may suggest a novel therapy for ROP in the future.

\section{Summary}

\section{What was known before}

- Maternal smoking during pregnancy has been causally associated with adverse pregnancy outcomes, including placental abruption, placenta previa, preterm delivery, intrauterine growth retardation, and foetal death.

What this study adds

- An inhibitory effect of maternal smoking against developing severe ROP was suggested.

\section{Conflict of interest}

The authors declare no conflict of interest.

\section{References}

1 Chen J, Smith LE. Retinopathy of prematurity. Angiogenesis 2007; 10: 133-140.

2 Binkhathlan AA, Almahmoud LA, Saleh MJ, Srungeri S. Retinopathy of prematurity in Saudi Arabia: incidence, risk factors, and the applicability of current screening criteria. Br J Ophthalmol 2008; 92(2): 167-169.

3 Friling R, Axer-Siegel R, Hersocovici Z, Weinberger D, Sirota L, Snir M. Retinopathy of prematurity in assisted versus natural conception and singleton versus multiple births. Ophthalmology 2007; 114(2): 321-324.

4 Akkoyun I, Oto S, Yilmaz G, Gurakan B, Tarcan A, Anuk D et al. Risk factors in the development of mild and severe retinopathy of prematurity. J AAPOS 2006; 10(5): 449-453.

5 Darlow BA, Hutchinson JL, Henderson-Smart DJ, Donoghue DA, Simpson JM, Evans NJ, Australian and New Zealand Neonatal Network. Prenatal risk factors for severe retinopathy of prematurity among very preterm infants of the Australian and New Zealand Neonatal Network. Pediatrics 2005; 115(4): 990-996.

6 Triche EW, Hossain N. Environmental factors implicated in the causation of adverse pregnancy outcome. Semin Perinatol 2007; 31: 240-242.

7 Shea AK, Steiner M. Cigarette smoking during pregnancy. Nicotine Tob Res 2008; 10(2): 267-278.

8 Leonardi-Bee JA, Smyth AR, Britton J, Coleman T. Environmental tobacco smoke on fetal health: Systematic review and meta-analysis. Arch Dis Child Fetal Neonatal Ed 2008; 93(5): F351-F361.

9 Jeppesen DL, Nielsen SD, Ersbøll AK, Valerius NH. Maternal smoking during pregnancy increases the risk of postnatal infections in preterm neonates. Neonatology 2008; 94(2): 75-78.

10 Sasaki S, Sata F, Katoh S, Saijo Y, Nakajima S, Washino N et al. Adverse birth outcomes associated with maternal smoking and polymorphisms in the N-nitrosaminemetabolizing enzyme genes NQO1 and CYP2E1. Am J Epidemiol 2008; 167(6): 719-726.

11 International Committee for the Classification of Retinopathy of Prematurity. The International Classification of Retinopathy of Prematurity revisited. Arch Ophthalmol 2005; 123(7): 991-999.

12 McGinnity FG, Halliday HL. Perinatal predictors of ocular morbidity in school children who were very low birthweight. Paediatr Perinat Epidemiol 1993; 7(4): 417-425.

13 Roy FH, Kelly ML. Chronic maternal cigaret smoking and infant retrolental fibroplasia: negative results. Ann Ophthalmol 1971; 3(7): 693-694.

14 Sonmez K, Drenser KA, Capone Jr A, Trese MT. Vitreous levels of stromal cell-derived factor 1 and vascular endothelial growth factor in patients with retinopathy of prematurity. Ophthalmology 2008; 115(6): 1065-1070.

15 Evereklioglu C, Ozkiriş A, Alaşehirli B, Sari I, Güldür E, Cengiz $\mathrm{B}$ et al. Effect of gestational nicotine treatment on newborn rat retina: a histopathological and morphometric analysis. Ophthalmic Physiol Opt 2003; 23(6): 527-533.

16 Sidle EH, Casselman R, Smith GN. Effect of cigarette smoke on placental antioxidant enzyme expression. Am J Physiol Regul Integr Comp Physiol 2007; 293(2): R754-R758.

17 Fayol L, Gulian JM, Dalmasso C, Calaf R, Simeoni U, Millet $\mathrm{V}$. Antioxidant status of neonates exposed in utero to tobacco smoke. Biol Neonate 2005; 87(2): 121-126. 